\title{
Presence of a High Flow-Mediated Constriction Phenomenon Prior to Flow-Mediated Dilation in Normal Weight, Overweight and Obese Children and Adolescents
}

\author{
Joseph D. Ostrem, M.S. ${ }^{a}$, Nicholas Evanoff, M.S. ${ }^{a}$, Aaron S. Kelly, Ph.D. ${ }^{b, c}$, and Donald R. \\ Dengel, Ph.D. $a, b$ \\ aLaboratory of Integrative Human Physiology, School of Kinesiology, University of Minnesota, \\ Minneapolis, Minnesota, 55455 \\ bDepartment of Pediatrics, University of Minnesota Medical School, Minneapolis, Minnesota, \\ 55455
}

'Department of Medicine, University of Minnesota Medical School, Minneapolis Minnesota 55455

\begin{abstract}
Purpose-Immediately following reactive hyperemia, the arteries in some individuals constrict before they begin to dilate. We have defined this physiological response high flow-mediated constriction (H-FMC). The aim of this study was to describe the frequency of the H-FMC during reactive hyperemia in children and adolescents throughout a range of body mass index (BMI) values and investigate differences in flow-mediated dilation (FMD), cardiovascular, and anthropometric measures between those subjects who experience this phenomenon and those who do not.
\end{abstract}

Methods-FMD was assessed in 136 children and adolescents ( 61 male, 75 female; $13 \pm 3$ years) by ultrasound imaging the brachial artery. H-FMC was characterized as the lowest point from the baseline brachial artery diameter immediately following reactive hyperemia cuff release.

Independent t-tests were used to compare differences between subjects who demonstrated an HFMC vs. Non-H-FMC.

Results-H-FMC was observed in 91 of the 136 participants (66.9\%). No significant difference was found between H-FMC vs. Non-H-FMC subjects for age ( $\mathrm{P}=0.602)$, gender $(\mathrm{P}=0.767)$, height $(\mathrm{P}=0.227)$ or weight $(\mathrm{P}=0.171)$. BMI percentile was trending toward significance in H-FMC vs. Non-H-FMC individuals $\left(91.8^{\text {th }} \pm 14.9^{\text {th }} v s .84 .6^{\text {th }} \pm 22.8^{\text {th }}\right.$ percentile, $\left.\mathrm{P}=0.057\right)$. FMD was significantly lower in H-FMC vs. Non-H-FMC subjects $(5.43 \pm 3.41$ vs. $8.05 \pm 3.97 \%, \mathrm{P}<0.001)$. If the H-FMC was added to the FMD there was no significant difference in dilation between H-FMC and Non-H-FMC individuals (8.03 $\pm 3.27 \%$ vs.8.05 $\pm 3.97 \%, \mathrm{P}=0.977)$.

Correspondence To: Joseph Ostrem, M.S., University of Minnesota, 1900 University Avenue S.E., 110 Cooke Hall, Minneapolis, MN 55455, Phone Number: (507) 272-3926, Fax Number: (612) 625-8867, ostre023@umn.edu.

Conflict of Interest: The authors have no conflicts of interest. 
Conclusion-Approximately $67 \%$ of participants demonstrated the high flow-mediated constriction during reactive hyperemia with BMI percentile being higher and FMD lower in children and adolescents who displayed this phenomenon.

\section{Keywords}

Ultrasound; Reactive Hyperemia; Flow Mediated Dilation; Flow Mediated Constriction; Adolescents

\section{Introduction}

Flow-mediated dilation (FMD) is widely used as a noninvasive method for measurement of endothelial function through ultrasound imaging of the brachial artery following reactive hyperemia. ${ }^{1}$ Endothelial dysfunction has been associated with cardiovascular events, ${ }^{2}$ as well as increasing age, ${ }^{3}$ gender, ${ }^{4,5}$ cigarette smoking, ${ }^{6}$ and obesity. ${ }^{7,8}$ An increase in shear stress via increased flow has been thought to initiate endothelial nitric oxide synthase (eNOS) production of $\mathrm{NO}^{9,10}$ A delayed dilatory response of an artery to shear stress has been observed in radial, ${ }^{11}$ brachial $^{1}$ and femoral ${ }^{12}$ vascular beds. The delayed dilation response of the radial artery to shear stress was investigated in a relatively recent study, ${ }^{11}$ which concluded that during high blood flow an opposing vasoconstrictor stimulus conceals a shear stress-induced NO-mediated dilation of the artery. It was postulated that vasoconstriction could result from a hemodynamic effect of increased blood flow leading to decreased transmural pressure and/or a shear stress-stimulated constrictor release, such as endothelin- $1 .{ }^{11,13}$

A delayed dilation response is regularly observed in humans; ${ }^{1,14-15}$ however, immediately following reactive hyperemia, the arteries of some individuals constrict before they begin to dilate. This physiological response has been referred to as a flow-mediated constriction (Figure 1). ${ }^{11,16-17,18}$ Alternative measures of cardiovascular response to FMD have been reported at the radial artery observing a constriction effect of the artery during distal occlusion. ${ }^{18}$ This procedure has been termed low-flow-mediated constriction (L-FMC). ${ }^{18}$ In contrast, a high-flow-mediated constriction (H-FMC) has been observed in healthy adults at the radial artery following occlusion. ${ }^{11}$ However, to our knowledge, no one has published on the occurrence of H-FMC observed at the brachial artery in children and adolescents. The aim of this study was to describe the frequency of the H-FMC during reactive hyperemia in children and adolescents throughout a large range of body mass index (BMI) values and to investigate differences in FMD, hemodynamic and anthropometric measures between those subjects who experience this phenomenon and those who do not.

\section{Materials and Methods}

\section{Study Population}

One hundred thirty-six children and adolescents (61 males, 75 females) were assessed for peak FMD. Subjects were recruited from a pediatric weight management clinic (patients and siblings) and from the community via flyers and advertisements. The study protocol was reviewed and approved by the University of Minnesota Institutional Review Board and all

J Clin Ultrasound. Author manuscript; available in PMC 2016 October 01. 
participants, along with parents/guardians, gave written informed assent and consent. The procedures followed in the study were in accordance with the institutional review board and HIPAA guidelines. Subjects were fasted for at least 8 hours prior to vascular assessment and were asked to abstain from caffeine for at least 4 hours on the morning of testing. Avoidance of strenuous exercise or physical activity was also required for 24 hours prior to the study visit.

\section{Physical Assessments}

Measurements for height and weight were obtained with a standard stadiometer (Avrton, Model S100, Prior Lake, MN, USA) and electronic scale (ST Scale-Tronix, Serial No. 5022-8893, White Plains, NY, USA), respectively. Body mass index (BMI) was calculated as weight in kilograms $(\mathrm{kg})$ divided by height in meters-squared $\left(\mathrm{m}^{2}\right)$. Tanner stage was assessed by a trained pediatrician or registered nurse. Waist and hip circumferences $(\mathrm{cm})$ were obtained with a Gulick measuring tape (Creative Health Products, Ann Arbor, MI, USA).

\section{Vascular Assessments}

Vascular testing was performed in the Vascular Biology Laboratory in the Clinical and Translation Science Institute (CTSI) at the University of Minnesota. Subjects were tested in a quiet, climate-controlled room $\left(22-23^{\circ} \mathrm{C}\right)$. Resting blood pressure was recorded using an automated sphygmomanometer (Colin Medical Instruments Corp., San Antonio, TX, USA) on the right arm prior to FMD assessment. A blood pressure cuff was also placed on the left forearm. Following 15 minutes of quiet rest in the supine position, vascular images of the left brachial artery were obtained proximal to the antecubital fossa in the longitudinal plane using a conventional ultrasound scanner (Acuson, Sequoia 512, Siemens Medical Solutions USA, Inc., Mountain View, CA, USA) with a 8-15 MHz linear array probe held at a constant pressure on the skin and at a fixed point over the imaged artery by a stereotactic arm.

The blood pressure cuff on the left forearm was inflated to a suprasystolic pressure level of $200 \mathrm{mmHg}$ and maintained for 5 minutes. Vascular images were captured 20-seconds prior to cuff release until 3 minutes post-cuff release and were digitized and stored on a personal computer for later off-line analysis using an electronic wall-tracking software program (Vascular Research Tools 5, Medical Imaging Application, LLC, Iowa City, IA, USA). Vascular images were assessed and baseline brachial artery measurements were recorded as a 10-second average just prior to blood pressure cuff release, i.e. occlusion baseline, and a 10 -second average immediately following blood pressure cuff release. Peak dilation during each study was defined as the 10-second average of the greatest percent change from baseline brachial artery diameter. Shear rate was used to estimate shear stress calculated as blood flow velocity $(q)$ divided by arterial diameter (D). Maximal flow $(\mathrm{m} / \mathrm{s})$ was used as a measure of average blood flow following cuff release. The high-flow-mediated constriction (H-FMC) was characterized using a 10s average of the lowest point from baseline brachial artery diameter following cuff release and considered present if the percent change was less than $-0.1 \%$ (Figure 1). A trained sonographer performed all digital ultrasound image 
analysis. When FMD was measured 7 days apart, the coefficient of variation was $11.1 \%$ in our laboratory, demonstrating good reproducibility.

\section{Statistical Analysis}

All statistical analysis was performed using IBM SPSS Statistics 21 (IBM Corp. Released 2012. IBM SPSS Statistics for Windows, Version 21.0. Armonk, NY: IBM Corp.).

Descriptive statistics were conducted for the two groups on anthropometric and hemodynamic measurements. Independent t-tests were used to compare differences between subjects who demonstrated an H-FMC vs. Non-H-FMC. A correlation matrix between analyzed parameters was implemented among significant anthropometric and vascular measures. A one-way analysis of variance (ANOVA) was conducted for the degree of $\mathrm{H}$ FMC for BMI percentile. An alpha value of 0.05 was denoted as statistically significant.

\section{Results}

Mean demographic characteristics of children are presented in Table 1. Of the 136 children and adolescents examined in this study, 91 (66.9\%) displayed an H-FMC. Children and adolescents ranged in age from 8 to 17 years old, with a mean age of $13 \pm 3$ years. When classified by BMI percentile, $70.6 \%$ of the study population was considered obese, $9.5 \%$ overweight and $19.9 \%$ were normal weight for their age and gender. When grouped by the presence or absence of H-FMC according to BMI percentile, $70.8 \%$ of obese and $76.9 \%$ of overweight children and adolescents displayed an H-FMC compared to $48.1 \%$ of normal weight children and adolescents displaying an H-FMC. Comparing BMI percentile with the H-FMC group and degree of H-FMC, average constriction was significantly greater in normal weight individuals than both overweight and obese combined $(-3.53 \pm 1.88 \%$ vs. $-2.45 \pm 1.59 \%, \mathrm{P}=0.03)$. No differences were observed for composition of gender, levels of systolic and diastolic blood pressure, heart rate, height, weight, BMI, Tanner stage, or hip and waist circumference (Table 1). BMI percentile trended towards being significantly higher in H-FMC than Non-H-FMC children and adolescents (Table 1). Table 2 displays the vascular measures for the study population. Baseline brachial artery diameter in H-FMC individuals was not significantly different from Non-H-FMC individuals. Average shear, peak shear and maximal flow were also not significantly different between H-FMC and Non-H-FMC children and adolescents. Peak FMD was significantly lower in H-FMC individuals compared to Non-H-FMC.

A correlation matrix was constructed using FMD, H-FMC, BMI and BMI percentile measures. A greater H-FMC was associated with lower peak FMD $(r=0.455, \mathrm{P}<0.001)$. Peak FMD was positively correlated with BMI $(r=0.243, P=0.004)$ and BMI percentile $(r=0.234$, $\mathrm{P}=0.006)$. A greater $\mathrm{H}-\mathrm{FMC}$ was not significantly correlated with increased $\mathrm{BMI}(\mathrm{r}=-0.006$, $\mathrm{P}=0.949$ ) or BMI percentile ( $\mathrm{r}=-0.066, \mathrm{P}=0.442)$. A one-way ANOVA for degree of H-FMC revealed no significant difference between or within groups based on BMI percentile ( $\mathrm{P}=0.92)$. 


\section{Discussion}

The aim of the study was to examine the prevalence of a flow-mediated constriction during reactive hyperemia in normal weight, overweight and obese children and adolescents as well as investigate potential differences of anthropometric and hemodynamic measures to $\mathrm{H}$ FMC presence. In the present study we observed that approximately $67 \%$ of the population displayed H-FMC. Those individuals that displayed H-FMC had a lower peak FMD compared to individuals that did not display an H-FMC.

Jiang et al. ${ }^{11}$ suggested two explanations for an observed constriction following cuff release: 1) a flow-mediated constriction factor or 2) a reduction in intra-arterial pressure generated by increased flow. An argument could be made that Non-H-FMC children and adolescents could possibly have a blunted shear-stimulated release of a constrictor agent, such as endothelin-1, ${ }^{11,13}$ compared to H-FMC children and adolescents. If this were true, one would expect there to be a difference in the time to peak between H-FMC and Non-H-FMC groups, which there is not. A blunted shear stress release of vasoconstrictors such as endothelin-1 could, theoretically, be due to a higher level of shear stress in Non-FMC before cuff release, as high levels of shear stress in cultured endothelial cells suppressed endothelin-1 release. ${ }^{13}$ However, in the present study there was not a significant difference in shear stress or maximal flow between those who displayed an H-FMC and those who did not display an H-FMC.

Another possible explanation may be that H-FMC children have a lower sensitivity to endothelin-1, such that for a given level of endothelin-1, less constriction occurs. Research by Weil et al. ${ }^{19}$ involving overweight and obese adults supports the notion that overweight and obesity are associated with enhanced endothelin-1 mediated vasoconstriction. Enhanced constriction could contribute to endothelial vasodilator dysfunction and increase risk of hypertension. In the present study, $85.7 \%$ of H-FMC children and adolescents were categorized as overweight or obese, while $68.8 \%$ of the Non-H-FMC children and adolescents met the classification. There was a trend for those demonstrating H-FMC to have a greater BMI percentile, which may support a flow-mediated constriction factor following cuff release. However, H-FMC and BMI percentile were not significantly correlated.

A significant drop in intra-arterial pressure may also be responsible for H-FMC. As blood flow is reestablished well above resting levels with removal of occlusion cuff pressure, a decrease in pressure could theoretically occur and cause the passive mechanical constriction of the artery. However, all things being equal, this response should have been observed in all subjects because the methodology was identical for H-FMC and Non-H-FMC children and adolescents. A significant difference between groups for shear stress and maximal flow might explain the presence of H-FMC, as children and adolescents with a greater shear would have greater blood flow following occlusion and potentially intra-arterial pressure. However, as stated in relation to endothelin-1 release, there was not a significant difference in shear stress or maximal flow between those who displayed an H-FMC and those who did not display an H-FMC. 
Regardless of whether H-FMC is caused by a flow-mediated constriction factor or a significant drop in intra-arterial pressure, children and adolescents with an H-FMC had a significantly lower FMD than the Non-H-FMC children and adolescents. Flow-mediated dilation has been utilized as a predictor of cardiovascular events in asymptomatic subjects. ${ }^{20}$ Overweight and obese children have displayed impaired FMD measures ${ }^{21}$ and are at increased risk for developing coronary heart disease risk factors. ${ }^{22}$ Higher FMD is generally associated with healthy endothelial function, and in the current study, Non-H-FMC children and adolescents displayed brachial artery FMD levels similar to levels of healthy control subjects in other studies ${ }^{23}$. Even though the presence of an H-FMC was associated with lower FMD response, it is unclear whether the H-FMC is associated with other cardiovascular risk factors as this will need to be examined in future studies.

Of interest, when an addition of the percent of H-FMC to peak FMD is applied to those displaying a vasoconstriction, the difference in peak FMD between the two groups disappears. It appears that there may be a discrete timeframe during which peak dilatory response to reactive hyperemia occurs. Therefore, if the initial response to cuff release immediately following reactive hyperemia is constriction, the result will lower the overall dilation of the brachial vessel if the initial baseline diameter is used. Statistical difference has been observed in the current study between the two groups for the composite end point and FMD measures (Table 2). H-FMC may have similar complementary value to FMD as LFMC, which was shown to have further value than a composite value (FMD+L-FMD) by reducing false-positive and false-negative results when used independently of FMD in clinical applications. ${ }^{24-25}$ Not only does the presence of an H-FMC complicate the analysis of FMD, but it also adds to the body of FMD research. The time course for dilation for both groups is similar, which seems to imply that NO and other dilating factors are secreted for a set amount of time in children and adolescents. Body mass index may also have an effect on the prevalence of vasoconstriction observed post-occlusion with H-FMC being a possible independent indicator of vascular change. Since obesity is often associated with endothelial dysfunction ${ }^{7,8}$, the presence of a H-FMC may be an initial sign of vascular dysfunction, as there is a moderate correlation between H-FMC and FMD $(r=0.455 ; \mathrm{P}<0.001)$ without a correlation with $\mathrm{BMI}(\mathrm{r}=-0.006, \mathrm{P}=0.949)$ or $\mathrm{BMI}$ percentile ( $\mathrm{r}=-0.066, \mathrm{P}=0.442)$. However, this hypothesis will need to be examined in future studies.

Previous research has investigated ancillary measures to flow-mediated dilation. Low-flowmediated constriction has been documented as a response of the radial artery during distal occlusion. ${ }^{18,25}$ This measure provides an accurate measure of resting arterial tone as it is a measure of the arterial response to resting shear stress. ${ }^{18,25}$ However, the technique reported for measuring L-FMC is vastly different compared to H-FMC. Weissgerber et al. ${ }^{26}$ reported that L-FMC was observed in the radial artery but not in the brachial artery of healthy pregnant and non-pregnant women. It would appear that L-FMC in the brachial artery is not as uniformed of a response as the radial artery; a significant increase in brachial artery diameter during cuff occlusion was observed in healthy children and young adults. ${ }^{27}$ Not only does the imaged vascular bed differ, but the time course of the constriction is chronologically different; L-FMC measuring constriction during blood flow restriction while $\mathrm{H}-\mathrm{FMC}$ is the constriction observed immediately following occlusion. Brachial artery $\mathrm{H}$ - 
FMC may have a comparable benefit on cardiovascular health assessment as L-FMC of the radial artery.

The present study is subject to a few limitations. Intra-arterial pressure and vasoconstrictor mediators were not directly measured. Since many of the participants were recruited from a pediatric weight management clinic (in addition to the community), the percentage of overweight and obese children in this study was much higher than in the general pediatric population. Future studies could investigate children with lower BMI values for a presence and frequency of an H-FMC as well as the prevalence of an H-FMC in differing adult populations. Investigation of health risk factors possibly associated with an H-FMC would also be beneficial. In addition, assessment of L-FMC would be beneficial when assessing FMD and H-FMC in future studies. A prospective study would be required to determine the validity and reliability of H-FMC as a risk factor for atherosclerosis and cardiovascular disease.

\section{Conclusion}

A high-flow-mediated constriction occurs in a majority of overweight and obese children and adolescents during reactive hyperemia and FMD was lower in children and adolescents who experience this phenomenon, suggesting the presence of endothelial dysfunction. Whether H-FMC has any clinical implications is currently unknown. However, our findings suggest that this phenomenon should be considered when analyzing and interpreting FMD data. Future studies should examine the relationship of the presence of H-FMC with cardiovascular risk factors in children and adults.

\section{Acknowledgments}

The authors wish to thank the participants for volunteering their time. The authors are grateful to Annie Sheldon and Cameron Naughton for coordinating the data collection for this study.

Funding Support: The project described was supported by Grant Number R01HL110957 from the National Heart, Lung, And Blood Institute (awarded to A.S.K). The content is solely the responsibility of the authors and does not necessarily represent the official views of the National Heart, Lung, And Blood Institute or the National Institutes of Health. Research reported in this publication was supported by the National Center for Advancing Translational Sciences of the National Institutes of Health Award Number UL1TR000114. The content is solely the responsibility of the authors and does not necessarily represent the official views of the National Institutes of Health.

\section{References}

1. Corretti MC, Anderson TJ, Benjamin EJ, et al. Guidelines for the ultrasound assessment of endothelial-dependent flow-mediated vasodilation of the brachial artery: a report of the International Brachial Artery Reactivity Task Force. J Am Coll Cardiol. 2002; 39:257. [PubMed: 11788217]

2. Deanfield JE, Halcox JP, Rabelink TJ. Endothelial function and dysfunction: testing and clinical relevance. Circulation. 2007; 115:1285. [PubMed: 17353456]

3. Celermajer DS, Sorensen KE, Spiegelhalter DJ, et al. Aging is associated with endothelial dysfunction in healthy men years before the age-related decline in women. JACC. 1994; 24:471. [PubMed: 8034885]

4. Kapuku GK, Treiber FA, Hartley B, et al. Gender influences endothelial-dependent arterial dilation via arterial size in youth. Am J Med Sci. 2004; 327:305. [PubMed: 15201641] 
5. Juonala M, Kahonen M, Laitinen T, et al. Effect of age and sex on carotid intima-media thickness, elasticity and brachial endothelial function in healthy adults: the cardiovascular risk in Young Finns Study. Eur Heart J. 2008; 29:1198. [PubMed: 18079136]

6. Lekakis J, Papamichael C, Vemmos C, et al. Effects of acute cigarette smoking on endotheliumdependent arterial dilation in normal subjects. Am J Cardiol. 1998; 81:1225. [PubMed: 9604954]

7. Tounian P, Aggoun Y, Dubern B, et al. Presence of increased stiffness of the common carotid artery and endothelial dysfunction in severely obese children: a prospective study. Lancet. 2001; 358:1400. [PubMed: 11705484]

8. Williams IL, Chowienczyk PJ, Wheatcroft SB, et al. Endothelial function and weight loss in obese humans. Obes Surg. 2005; 15:1055. [PubMed: 16105407]

9. Corson MA, James NL, Latta SE, et al. Phosphorylation of endothelial nitric oxide synthase in response to fluid shear stress. Circ Res. 1996; 79:984. [PubMed: 8888690]

10. Pyke KE, Tschakovsky ME. The relationship between shear stress and flow-mediated dilatation: implications for the assessment of endothelial function. J Physiol. 2005; 568:357. [PubMed: 16051630]

11. Jiang B, Seddon M, Fok H, et al. Flow-mediated dilation of the radial artery is offset by flowinduced reduction in transmural pressure. Hypertension. 2011; 57:1145. [PubMed: 21502570]

12. Kooijman M, Thijssen DHJ, de Groot PCE, et al. Flow-mediated dilatation in the superficial femoral artery is nitric oxide mediated in humans. J Physiol. 2008; 586:1137. [PubMed: 18096601]

13. Kuchan MJ, Frangos JA. Shear stress regulates endothelin-1 release via protein kinase $\mathrm{C}$ and cGMP in cultured endothelial cells. Am J Physiol. 1993; 264:H150. [PubMed: 8381608]

14. Corretti MC, Plotnick GD, Vogel RA. Technical aspects of evaluating brachial artery vasodilatation using high-frequency ultrasound. Am J Physiol. 1995; 268:H1397. [PubMed: 7733339]

15. Black MA, Cable NT, Thijssen DHJ, et al. Importance of measuring the time course of flowmediated dilatation in humans. Hypertension. 2008; 51:203. [PubMed: 18086954]

16. Rubanyi, Gabor M. Flow-induced Vasodilation of Large Arteries from "Ascending Reflex" to EDRF Flow-Dependent Regulation of Vascular Function. New York: Springer; 1995. p. 119

17. Turner MJ. Is pressure decrease at peak hyperemia attributed to Poiseulle or Bernoulli or both? Hypertension. 2011; 58:e21. [PubMed: 21844491]

18. Gori T, Gragoni S, Lisi M, et al. Conduit artery constriction mediated by low flow a novel noninvasive method for the assessment of vascular function. JACC. 2008; 51:1953. [PubMed: 18482663]

19. Weil BR, Westby CM, Van Guilder GP, et al. Enhanced endothelin-1 system activity with overweight and obesity. Am J Physiol Circ Physiol. 2011; 301:H689.

20. Thijssen DH, Black MA, Pyke KE, et al. Assessment of flow-mediated dilation in humans: a methodological and physiological guideline. Am J Physiol Heart Circ Physiol. 2011; 300:H2. [PubMed: 20952670]

21. Meyer AA, Kundt G, Steiner M, et al. Impaired flow-mediated vasodilation, carotid artery intimamedia thickening, and elevated endothelial plasma markers in obese children: the impact of cardiovascular risk factors. Pediatrics. 2006; 117:1560. [PubMed: 16651309]

22. Freedman DS, Dietz WH, Srinivasan SR, et al. The relation of overweight to cardiovascular risk factors among children and adolescents: the Bogalusa Heart Study. Pediatrics. 1999; 103:1175. [PubMed: 10353925]

23. Babar GS, Zidan H, Widlansky ME, et al. Impaired endothelial function in preadolescent children with type 1 diabetes. Diabetes Care. 2011; 34:681. [PubMed: 21289230]

24. Humphreys RE, Green DJ, Cable NT, et al. Low-flow mediated constriction: the yin to FMD's yang? Expert Rev Cardiovasc Ther. 2014; 12:557. [PubMed: 24745959]

25. Gori T, Grotti S, Dragoni S, et al. Assessment of vascular function: flow-mediated constriction complements the information of flow-mediated dilatation. Heart. 2010; 96:141. [PubMed: 19858140] 
26. Weissgerber TL, Davies GA, Tschakovsky ME, et al. Low-flow mediated constriction occurs in the radial but not the brachial artery in healthy pregnant and nonpregnant women. J Appl Physiol. 2010; 108:1097. [PubMed: 20185630]

27. Thijssen DHJ, van Bemmel MM, Bullens LM, et al. The impact of baseline diameter on flowmediated dilation differs in young and older humans. Am J Physiol Heart Circ Physiol. 2008; 295:H1594. [PubMed: 18708443] 

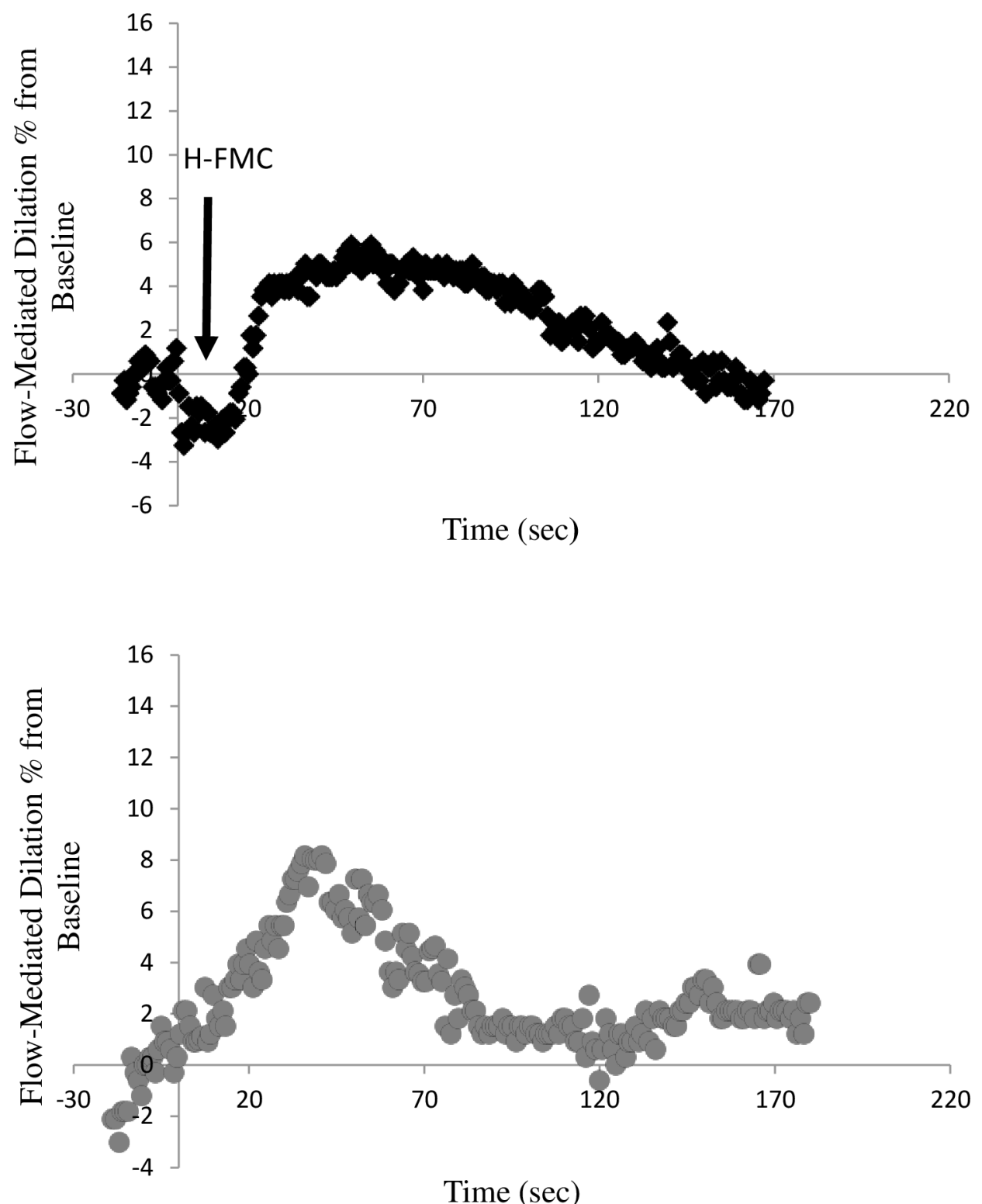

Figure 1.

A representation of a Flow-Mediated Dilation (percent change in brachial artery diameter from baseline) response in H-FMC $(\bullet)$ and Non-H-FMC $(\bullet)$ children. 


\section{Table 1}

\section{Demographic Characteristics}

\begin{tabular}{llll}
\hline & H-FMC & Non-H-FMC & P-value \\
\hline$n(\%$ male $)$ & $91(44 \%)$ & $45(47 \%)$ & \\
Age, years & $13.0 \pm 2.5$ & $12.8 \pm 2.7$ & 0.602 \\
Blood pressure, mmHg & & & \\
Systolic & $118 \pm 12$ & $117 \pm 13$ & 0.849 \\
Diastolic & $61 \pm 9$ & $60 \pm 8$ & 0.480 \\
Height, cm & $158.8 \pm 12.1$ & $155.9 \pm 14.5$ & 0.227 \\
Weight, kg & $76.9 \pm 26.4$ & $70.3 \pm 26.3$ & 0.171 \\
Tanner Stage & $3 \pm 1$ & $3 \pm 1$ & 0.477 \\
Hip Circumference, cm & $100.5 \pm 16.3$ & $97.4 \pm 17.4$ & 0.308 \\
Waist Circumference, cm & $87.2 \pm 16.7$ & $83.5 \pm 17.8$ & 0.235 \\
Body Mass Index, $\mathrm{kg} / \mathrm{m}^{2}$ & $29.8 \pm 7.5$ & $27.8 \pm 7.8$ & 0.151 \\
Body Mass Index Percentile, & $91.8 \pm 14.9$ & $84.6 \pm 22.8$ & 0.057 \\
\hline
\end{tabular}

Note: Values are means $\pm \mathrm{SD} ; n$, number of participants/group.

H-FMC - High-Flow Mediated Constriction

Non-FMC - No High-Flow Mediated Constriction 
Table 2

\section{Measures of vascular function}

\begin{tabular}{|c|c|c|c|}
\hline & H-FMC & Non-H-FMC & P-value \\
\hline Brachial Artery Diameter, mm & $3.33 \pm 0.44$ & $3.24 \pm 0.40$ & 0.296 \\
\hline Time to Peak FMD, sec & $59.0 \pm 24.3$ & $62.1 \pm 34.3$ & 0.591 \\
\hline Average Shear, $\sec ^{-1}$ & $262.5 \pm 62.1$ & $273.7 \pm 61.1$ & 0.321 \\
\hline Peak Shear, $\sec ^{-1}$ & $294.4 \pm 74.6$ & $301.5 \pm 65.6$ & 0.589 \\
\hline Maximal Flow, m/s & $0.85 \pm 0.18$ & $0.89 \pm 0.17$ & 0.168 \\
\hline Brachial Artery Peak Diameter, mm & $3.56 \pm 0.47$ & $3.55 \pm 0.40$ & 0.826 \\
\hline Flow-Mediated Dilation, $\%$ & $5.43 \pm 3.41$ & $8.05 \pm 3.97$ & $<0.001$ \\
\hline $\mathrm{H}-\mathrm{FMC}+\mathrm{FMD}, \%$ & $8.03 \pm 3.27$ & $8.05 \pm 3.97^{*}$ & 0.977 \\
\hline
\end{tabular}

Note: Values are means \pm SD;

* Non-H-FMC is the same for FMD and H-FMC+FMD

Maximal flow $(\mathrm{m} / \mathrm{s})$ is a measure of average blood flow following cuff release.

FMD - Flow Mediated Dilation

H-FMC - High-Flow Mediated Constriction

Non-FMC - No High-Flow Mediated Constriction 